\title{
Phase Diagram of Quark Matter in Nonlocal Chiral Models under Color and Electric Charge Neutrality Conditions
}

\author{
D. Gómez Dumm ${ }^{a, b}$, A. G. Grunfeld ${ }^{c}$, and N. N. Scoccola ${ }^{b, c, d}$ \\ ${ }^{a}$ IFLP, CONICET - Dpto. de Física, Universidad Nacional de La Plata, C.C. 67, 1900 La Plata, Argentina \\ ${ }^{b}$ CONICET, Rivadavia 1917, 1033 Buenos Aires, Argentina \\ c Physics Department, Comisión Nacional de Energía Atómica, \\ Av. Libertador 8250, 1429 Buenos Aires, Argentina and \\ ${ }^{d}$ Universidad Favaloro, Solís 453, 1078 Buenos Aires, Argentina
}

Received on 5 October, 2006

\begin{abstract}
We consider the phase diagram of two-flavor quark matter under neutron star constraints for the case of two nonlocal, covariant quark models within the mean field approximation. In one of these models (Model I) the nonlocality arises from the regularization procedure, motivated by the instanton liquid model, whereas in the second one (Model II) a separable approximation of the one-gluon exchange interaction is applied. We find that Model II predicts a larger quark mass gap, and the corresponding critical temperature at $\mu=0, T_{c}(0) \simeq 140$ $\mathrm{MeV}$, is in better agreement with recent lattice QCD results than the prediction of the standard local NJL model, which exceeds $200 \mathrm{MeV}$. For both models we have considered various coupling strengths in the scalar diquark channel, showing that different low-temperature quark matter phases can occur at intermediate densities: a normal quark matter (NQM) phase, a superconducting quark matter (2SC) phase and a mixed 2SC-NQM phase. In most cases, a narrow gapless $2 \mathrm{SC}$ phase region is also obtained at finite temperatures.
\end{abstract}

Keywords: Quark matter; Neutron stars; High density

\section{INTRODUCTION}

Within the last decade the investigation of the thermodynamics and phase structure of strongly interacting matter has been driven by the results of the experimental programs with ultrarelativistic heavy ion beams at CERN-SPS and BNLRHIC, as well as by the unprecedented quality of data from lattice QCD simulations. A new picture of the state of matter created in these experiments has emerged, according to which the physical nature of the sought-for quark-gluon plasma (QGP) is a perfect liquid of strongly correlated hadron-like resonances rather than an ideal gas of quasifree quarks and gluons [1]. Turning to the domain of finite chemical potentials, guidance from lattice QCD is limited to $\mu \leq T$ (where Taylor expansion techniques can be applied), and experimental programmes such as CBM at FAIR Darmstadt are still in a first stage. Therefore, predictions for the QCD thermodynamics at low temperatures and high baryon densities have to be developed within effective models for strongly coupled, nonperturbative QCD, to be tested against observational constraints from neutron stars [2].

For two-flavor isospin symmetric quark matter the QCD phase diagram has been explored in the frame of different quark models. All of them agree in finding that a two-flavor color superconducting phase (2SC) occurs at low temperatures and moderate chemical potentials [3]. However, when one considers different chemical potentials for each quark flavor and color, and compact star conditions are imposed -i.e., color and electric charge neutrality conditions, together with $\beta$ equilibrium-, the situation is more complicated and different models lead to qualitatively different results. For example, it has been claimed [4] that in the interior of nucleon stars the 2SC phase would be disfavored against the color-flavorlocking (CFL) one. In contrast, results from models with self- consistently determined quark masses $[5,6]$ indicate that the 2SC phase is favored in an intermediate regime of densities, before strange quarks become relevant. There are also results arising from a noncovariant nonlocal quark model [7] which show that the 2SC phase is not present in asymmetric quark matter for standard values of the diquark coupling if one assumes Gaussian regulator functions [8]. In this model, for strong diquark couplings one does find a 2SC phase, together with a gapless $2 \mathrm{SC}$ (g2SC) and a "mixed phase" region in which normal quark matter (NQM) and 2SC phases coexist.

Given these qualitatively different results, it is important to investigate the situation in the case of effective models that go beyond those used in Refs. [4, 6, 8], in the sense that they include fully covariant nonlocal interactions. Nonlocality arises naturally in the context of several successful approaches to low energy quark dynamics, such as the instanton liquid model [9] and the Schwinger-Dyson resummation techniques [10]. The same happens in lattice QCD [11]. It is also argued that nonlocal models have several advantages over the local ones (e.g., the Nambu-Jona-Lasinio model [12] and its generalizations). Indeed, nonlocal interactions regularize the models in such a way that anomalies are preserved [13] and charges properly quantized, the effective interaction is finite to all orders in the loop expansion and there is no need to introduce extra cut-offs, soft regulators lead to small nextto-leading order corrections [14], etc. This type of models has been successfully used to investigate meson [15-18] and baryon [19] properties at vanishing temperature and chemical potential, and the phase diagram of isospin symmetric matter has also been studied within this context [20-23]. Now our aim is to extend these analyses to the case in which compact star conditions are imposed.

The article is organized as follows. In Sect. II we introduce the model under consideration. Then, in Sect. III we present the numerical results obtained for the case of a Gaussian reg- 
ulator, considering different ratios between the coupling constants. In Sect. IV we discuss the features of the obtained phase diagrams, while in Sect. V we state our conclusions.

\section{FORMALISM}

Let us begin by stating the Euclidean action for the nonlocal chiral quark model in the case of two light flavors and antitriplet diquark interactions,

$$
\begin{aligned}
S_{E}= & \int d^{4} x\{\bar{\psi}(x)(-i \partial+m) \psi(x) \\
& \left.-\frac{G}{2} j_{M}^{f}(x) j_{M}^{f}(x)-\frac{H}{2}\left[j_{D}^{a}(x)\right]^{\dagger} j_{D}^{a}(x)\right\} .
\end{aligned}
$$

Here $m$ is the current quark mass, which is assumed to be equal for $u$ and $d$ quarks. The nonlocality can be introduced now in different ways [24]. In what follows we will work within two alternative scenarios, that we call "Model I" and "Model II", in which the currents $j_{M, D}(x)$ in Eq.(1) are given by nonlocal operators. In the case of Model I [16, 17], the effective interactions are based in an instanton liquid picture of QCD. The nonlocal currents read

$$
\begin{aligned}
& j_{M}^{f}(x)=\int d^{4} y d^{4} z r(y-x) r(x-z) \bar{\psi}(y) \Gamma_{f} \psi(z), \\
& j_{D}^{a}(x)=\int d^{4} y d^{4} z r(y-x) r(x-z) \bar{\psi}_{C}(y) i \gamma_{5} \tau_{2} \lambda_{a} \psi(z),
\end{aligned}
$$

where we have defined $\psi_{C}(x)=\gamma_{2} \gamma_{4} \bar{\psi}^{T}(x)$ and $\Gamma_{f}=\left(\mathbb{1}, i \gamma_{5} \vec{\tau}\right)$, while $\vec{\tau}$ and $\lambda_{a}$, with $a=2,5,7$, stand for Pauli and Gell-Mann matrices acting on flavor and color spaces, respectively.

On the other hand, Model II [15, 25] arises from a separable form of the effective one-gluon exchange picture. In this case the nonlocal currents $j_{M, D}(x)$ are given by

$$
\begin{aligned}
& j_{M}^{f}(x)=\int d^{4} z g(z) \bar{\psi}\left(x+\frac{z}{2}\right) \Gamma_{f} \psi\left(x-\frac{z}{2}\right), \\
& j_{D}^{a}(x)=\int d^{4} z g(z) \bar{\psi}\left(x+\frac{z}{2}\right) i \gamma_{5} \tau_{2} \lambda_{a} \psi\left(x-\frac{z}{2}\right) .
\end{aligned}
$$

The functions $r(x-y)$ and $g(z)$ in Eqs. (2) and (3) are nonlocal regulators characterizing the corresponding interactions.

The effective action in Eq. (1) might arise via Fierz rearrangement from some underlying more fundamental interactions, and is understood to be used - at the mean field level — in the Hartree approximation. In general, the ratio of

$$
S^{-1}=\left(\begin{array}{cccccccc}
-p_{u r}^{+}+\Sigma_{u r} & 0 & 0 & 0 & 0 & 0 & \gamma_{5} \tau_{2}^{c} \Delta & 0 \\
0 & -p_{u b}^{+}+\Sigma_{u b} & 0 & 0 & 0 & 0 & 0 & 0 \\
0 & 0 & -p_{d r}^{+}+\Sigma_{d r} & 0 & -\gamma_{5} \tau_{2}^{c} \Delta^{*} & 0 & 0 & 0 \\
0 & 0 & 0 & -p_{d b}^{+}+\Sigma_{d b} & 0 & 0 & 0 & 0 \\
0 & 0 & \gamma_{5} \tau_{2}^{c} \Delta^{*} & 0 & -p_{u r}^{-}+\Sigma_{u r} r^{*} & 0 & 0 & 0 \\
0 & 0 & 0 & 0 & 0 & -p_{u b}^{-}+\Sigma_{u b}{ }^{*} & 0 & 0 \\
-\gamma_{5} \tau_{2}^{c} \Delta & 0 & 0 & 0 & 0 & 0 & -p_{d r}^{-}+\Sigma_{d r}^{*} & 0 \\
0 & 0 & 0 & 0 & 0 & 0 & 0 & -p_{d b}^{-}+\Sigma_{d b}^{*}
\end{array}\right)
$$

Here the inverse propagator $S^{-1}(\bar{\sigma}, \bar{\Delta})$ is a $48 \times 48$ matrix in Dirac, flavor, color and Nambu-Gorkov spaces, given by

where the Euclidean action is obtained from Eq. (1) by going to momentum space and performing the replacements

$$
p_{4} \rightarrow \omega_{n}-i \mu_{f c}, \quad \int \frac{d^{4} p}{(2 \pi)^{4}} \rightarrow T \sum_{n=-\infty}^{\infty} \int \frac{d^{3} \vec{p}}{(2 \pi)^{3}}
$$

Here $p_{4}$ is the fourth component of the (Euclidean) momentum of a quark carrying flavor $f$ and color $c$, and $\omega_{n}$ are the Matsubara frequencies corresponding to fermionic modes, $\omega_{n}=(2 n+1) \pi T$ (we are assuming that quark interactions depend on $T$ and $\mu_{f c}$ only through the arguments of the regulators). Notice that we have introduced different chemical potentials for each quark flavor and color.

To proceed it is convenient to perform a standard bosonization of the theory. Thus, we introduce the bosonic fields $\sigma, \pi_{a}$ and $\Delta_{a}$, and integrate out the quark fields. In what follows we work within the mean field approximation (MFA), in which these bosonic fields are replaced by their vacuum expectation values $\bar{\pi}_{a}=0, \bar{\sigma}$ and $\bar{\Delta}_{a}$. Moreover, we adopt the usual 2SC ansatz $\bar{\Delta}_{5}=\bar{\Delta}_{7}=0, \bar{\Delta}_{2}=\bar{\Delta}$. Within this approximation, and employing the Nambu-Gorkov formalism, the mean field thermodynamical potential per unit volume can be written as

$$
\begin{aligned}
\Omega^{M F A} & =-\frac{T}{V} \ln Z^{M F A}= \\
& =\frac{\bar{\sigma}^{2}}{2 G}+\frac{|\bar{\Delta}|^{2}}{2 H}-\frac{T}{2} \sum_{n=-\infty}^{\infty} \int \frac{d^{3} \vec{p}}{(2 \pi)^{3}} \ln \operatorname{det}\left[\frac{S^{-1}}{T}\right] .
\end{aligned}
$$

coupling constants $H / G$ would be determined by these micro-
scopic couplings; for example, OGE interactions, as well as instanton model interactions, lead to $H / G=0.75$. However, since the precise derivation of the effective couplings from QCD is not known, there is a significant theoretical uncertainty in this value. In fact, so far there is no strong phenomlarger that $H / G \sim 1$ are quite unlikely to be realized in QCD, since they might lead to color symmetry breaking in the vacuum. We will leave the ratio as a free parameter, analyzing the

The partition function of the system at temperature $T$ and quark chemical potentials $\mu_{f c}$ is given by

$$
Z=\int D \bar{\psi} D \psi e^{-S_{E}\left(\mu_{f c}, T\right)}
$$


where we have used the definitions

$$
\begin{aligned}
p_{f c}^{ \pm} & =\left(\omega_{n} \mp i \mu_{f c}, \vec{p}\right), \\
\Sigma_{f c} & =m+\bar{\sigma} h\left(p_{f c}^{+}, p_{f c}^{+}\right), \\
\Delta & =\bar{\Delta} h\left(p_{u r}^{+}, p_{d r}^{-}\right),
\end{aligned}
$$

with $f=u, d$ and $c=r, g, b$. The functions $h(p, q)$ have been introduced in order to have a common notation for both Model I and Model II. One has

$$
h(s, t)=\left\{\begin{array}{l}
r\left(s^{2}\right) r\left(t^{2}\right) \\
g\left(\left[\frac{s+t}{2}\right]^{2}\right)
\end{array}\right.
$$

We have taken into account that, as we will see below, the usual 2SC ansatz implies $\mu_{f r}=\mu_{f g}$. Thus, in Eq. (7), entries with subindices $u r$ and $d r$ are intended to be multiplied by an $\mathbb{1}_{2 \times 2}$ matrix in $r g$ space, while $\tau_{2}^{c}$ stands for a $\tau_{2}$ Pauli matrix acting in this space.

For finite values of the current quark mass, $\Omega^{M F A}$ turns out to be divergent. We regularize it by defining

$$
\Omega_{(\mathrm{reg})}^{M F A}=\Omega^{M F A}-\Omega^{\text {free }}+\Omega_{(\text {reg })}^{\text {free }},
$$

where $\Omega^{\text {free }}$ is obtained from Eq. (6) by setting $\bar{\Delta}=\bar{\sigma}=0$, and $\Omega_{(\text {reg })}^{\text {free }}$ is the usual regularized form for the thermodynamical potential of a free fermion gas. Now the mean field values $\bar{\sigma}$ and $\bar{\Delta}$ can be obtained from the coupled gap equations

$$
\frac{d \Omega_{(\mathrm{reg})}^{M F A}}{d \bar{\Delta}}=0, \quad \frac{d \Omega_{(\mathrm{reg})}^{M F A}}{d \bar{\sigma}}=0 .
$$

So far we have introduced different chemical potentials for each quark flavor and color. When the system is in chemical equilibrium, not all of them are independent. Within the previously introduced 2SC ansatz, only one color-dependent chemical potential is needed to ensure color charge neutrality, and the $\mu_{f c}$ can be written in terms of only three independent quantities: the baryonic chemical potential $\mu_{B}$, the quark electric chemical potential $\mu_{Q_{q}}$ and the color chemical potential $\mu_{8}$. Defining $\mu \equiv \mu_{B} / 3$, the corresponding relations read

$$
\begin{aligned}
\mu_{u r}=\mu_{u g} & =\mu+\frac{2}{3} \mu_{Q_{q}}+\frac{1}{3} \mu_{8} \\
\mu_{d r}=\mu_{d g} & =\mu-\frac{1}{3} \mu_{Q_{q}}+\frac{1}{3} \mu_{8} \\
\mu_{u b} & =\mu+\frac{2}{3} \mu_{Q_{q}}-\frac{2}{3} \mu_{8} \\
\mu_{d b} & =\mu-\frac{1}{3} \mu_{Q_{q}}-\frac{2}{3} \mu_{8} .
\end{aligned}
$$

Now, in the core of neutron stars, in addition to quark matter we have electrons. Thus, within the mean field approximation for the quark matter, and considering the electrons as a free Dirac gas, the full grand canonical potential is given by

$$
\Omega^{\text {full }}=\Omega_{(\mathrm{reg})}^{M F A}+\Omega^{e},
$$

where

$$
\Omega^{e}=-\frac{1}{12 \pi^{2}}\left(\mu_{e}^{4}+2 \pi^{2} T^{2} \mu_{e}^{2}+\frac{7 \pi^{4}}{15} T^{4}\right)
$$

$\mu_{e}$ being the electron chemical potential (for simplicity we have neglected here the electron mass).

In addition, it is necessary to take into account that quark matter has to be in beta equilibrium with electrons through the beta decay reaction

$$
d \rightarrow u+e+\overline{\mathrm{v}}_{e}
$$

Thus, assuming that antineutrinos escape from the stellar core, we must have

$$
\mu_{d c}-\mu_{u c}=-\mu_{Q_{q}}=\mu_{e} .
$$

If we now require the system to be electric and color charge neutral, the number of independent chemical potentials reduces further: $\mu_{e}$ and $\mu_{8}$ are fixed by the conditions of vanishing electric and color densities, i.e.

$$
\begin{aligned}
\rho_{Q_{t o t}} & =\rho_{Q_{q}}-\rho_{e}=\sum_{c=r, g, b}\left(\frac{2}{3} \rho_{u c}-\frac{1}{3} \rho_{d c}\right)-\rho_{e}=0 \\
\rho_{8} & =\frac{1}{\sqrt{3}} \sum_{f=u, d}\left(\rho_{f r}+\rho_{f g}-2 \rho_{f b}\right)=0,
\end{aligned}
$$

where

$$
\rho_{e}=-\frac{\partial \Omega}{\partial \mu_{e}}=-\frac{\partial \Omega^{e}}{\partial \mu_{e}}, \rho_{f c}=-\frac{\partial \Omega}{\partial \mu_{f c}}=-\frac{\partial \Omega_{(\mathrm{reg})}^{M F A}}{\partial \mu_{f c}} .
$$

Consequently, in the physical situation we are interested in, for each value of $T$ and $\mu$ we should find the values of $\bar{\Delta}$, $\bar{\sigma}, \mu_{e}$ and $\mu_{8}$ that solve Eqs. (13), supplemented by Eqs. (18) and (19).

\section{NUMERICAL RESULTS}

In this section we present our numerical results, showing the features of the phase diagrams and the behavior of relevant physical quantities for Models I and II. According to previous analyses carried out within nonlocal scenarios [22], the results are not expected to show a strong qualitative dependence on the shape of the regulator. Thus we will concentrate here on simple and well-behaved Gaussian regulator functions, taking (in momentum space)

$$
\begin{aligned}
& r\left(p^{2}\right)=\exp \left(-p^{2} / 2 \Lambda^{2}\right) \\
& g\left(p^{2}\right)=\exp \left(-p^{2} / \Lambda^{2}\right)
\end{aligned}
$$

Here $\Lambda$ is a free model parameter, playing the rôle of an ultraviolet cut-off. We have chosen a different normalization for Models I and II in view of the relation between the respective regulating functions [see Eq. (11)], which determine the low $T$ and $\mu$ phenomenology. 


\section{A. Parameterization}

For definiteness, for both Models I and II we choose here input parameters $m, \Lambda$ and $G$ which allow to reproduce the empirical values for the pion mass $m_{\pi}=139 \mathrm{MeV}$ and decay constant $f_{\pi}=92.4 \mathrm{MeV}$, and lead to a phenomenologically acceptable value for the chiral condensates at vanishing $T$ and $\mu_{f c}$. For Gaussian regulators, taking into account the chosen normalization of the cut-offs, it is seen that within the MFA both models lead to the same expressions for the considered physical quantities at $T=\mu=0$. However, this is not the case when one goes beyond the MFA. In particular, the expressions for the pion mass and decay constant are different, therefore different sets of input parameters have to be used. The parameters considered here for Model I are $m=5.14 \mathrm{MeV}, \Lambda=971 \mathrm{MeV}$ and $G \Lambda^{2}=15.41$, while for Model II we have taken $m=5.12 \mathrm{MeV}, \Lambda=827 \mathrm{MeV}$ and $G \Lambda^{2}=18.78$. With these sets we get for both models a phenomenologically reasonable value for the chiral condensate, namely $\langle 0|\bar{q} q| 0\rangle^{1 / 3}=-250 \mathrm{MeV}$. The remaining free parameter is the coupling strength $H$ in the scalar diquark channel. In view of the difficulty of fixing this parameter from hadron phenomenology at zero $T$ and $\mu$, we study here the dependence of the results with $H$ by choosing different values for the coupling ratio $H / G$ in the range from 0.5 to 1 .

\section{B. Order parameters and phase transitions}

For fixed values of the temperature $T$ and the chemical potential $\mu$, the mean field values $\bar{\sigma}$ and $\bar{\Delta}$, as well as the chemical potentials $\mu_{e}$ and $\mu_{8}$, can be numerically obtained from the gap equations (13), together with the neutron star constraints (18) and (19). Let us begin by considering the case $H / G=0.75$, which is motivated by various effective models of quark-quark interactions. Our results for $\bar{\sigma}, \bar{\Delta}, \mu_{e}$ and $\mu_{8}$ are shown in Fig. 1, where we plot these quantities as functions of $\mu$ for different representative values of the temperature. Left and right panels correspond to Models I and II, respectively. For $T=0$ (solid lines), at low chemical potentials the system is in both cases in a chiral symmetry broken phase (CSB), where quarks acquire large dynamical masses. By increasing the chemical potential one reaches a first order phase transition in which the chiral symmetry is approximately restored, and the system gets into a phase which is not homogeneous but favors the coexistence of a 2SC phase and a normal quark matter (NQM) phase. In this mixed phase zone the electric neutrality is realized globally: the different phases have opposite electric charges which cancel each other, with a common equilibrium pressure. The chemical potential $\mu_{e}$, which for $T=0$ vanishes in the CSB phase but is nonzero in the mixed phase, also shows a discontinuity across the transition. As expected, the growth of the color chemical potential $\mu_{8}$ in the 2SC component of the mixed phase is approximately proportional to that of the corresponding $\bar{\Delta}$, which governs the amount of breakdown of the color symmetry due to quark pairing. When the temperature is increased (see dashed curves in Fig. 1, corresponding to $T=40 \mathrm{MeV}$ ), this mixed phase is
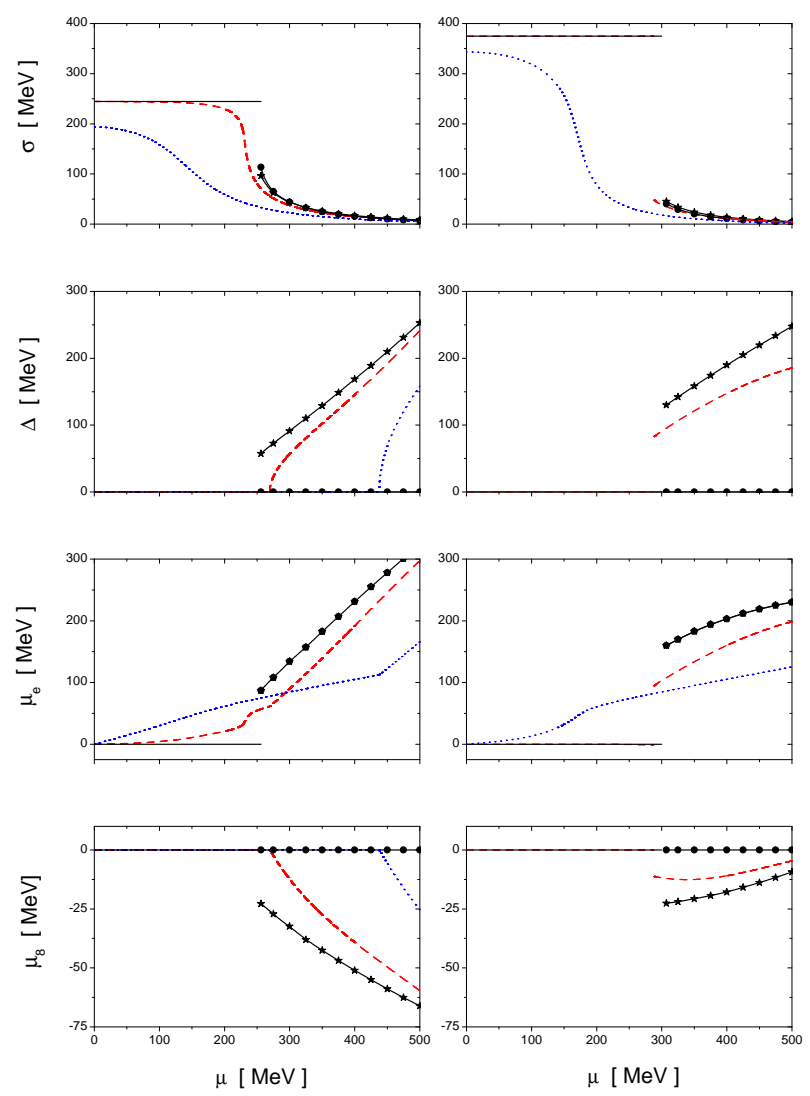

FIG. 1: Behavior of the mean fields $\bar{\sigma}$ and $\bar{\Delta}$ and the chemical potentials $\mu_{e}$ and $\mu_{8}$ for Models I (left) and II (right) as a function of the chemical potential, for three different values of the temperature. The curves correspond to the case $H / G=3 / 4$. Full lines correspond to $T=0$, dashed lines to $T=40 \mathrm{MeV}$ and dotted lines to $T=100 \mathrm{MeV}$. In the case of $T=0$, lines marked with stars and dots correspond to the $2 \mathrm{SC}$ and NQM phases respectively.

no longer favored and the system goes into a pure 2SC phase. For $T=40 \mathrm{MeV}$, this shows up as a second order transition in the case of Model I, and a first order transition in the case of Model II. Now, for both models, when one moves along the first order transition line from $T=0$ towards higher temperatures, one arrives at a triple point (3P). At this point the CSB and 2SC phases coexist with a third, normal quark matter (NQM) phase, in which the chiral symmetry is approximately restored and there is no color superconductivity. Finally, if $T$ is still increased, one reaches an "end point" (EP) where the first order transition from CSB to NQM phases becomes a smooth crossover. The behavior of the dynamical masses and the electric chemical potential $\mu_{e}$ along this smooth transition is shown in Fig. 1, see curves corresponding to $T=40 \mathrm{MeV}$ (Model I) and $T=100 \mathrm{MeV}$ (dotted lines, Models I and II).

\section{Quark matter phase diagrams}

The described features of the phase diagrams for Models I and II can be visualized in the graphs shown in Fig. 2, where 

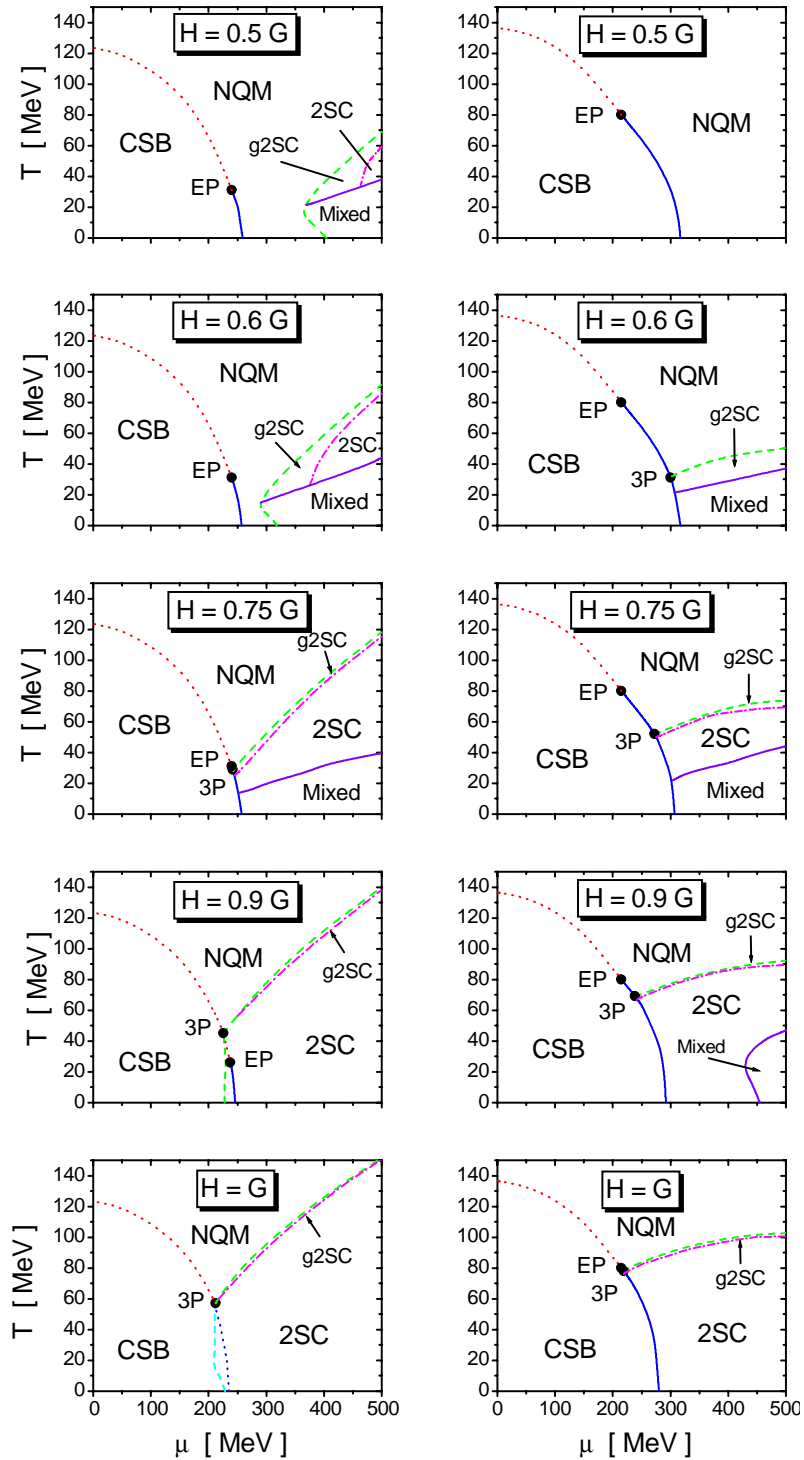

FIG. 2: Phase diagrams for Models I (left) and II (right) for different values of $H / G$. Full and dashed lines indicate first and second order phase transition curves respectively, dotted lines correspond to crossover-like transitions, and dashed lines delimit the gapless 2SC band. Different phases are denoted as NQM (normal quark matter phase), CSB (chiral symmetry broken phase) and 2SC (two-flavor superconducting phase), while the regions marked as "Mixed" correspond to the NQM-2SC mixed phase. EP and 3P denote the end points and triple points respectively.

we plot the transition curves on $T-\mu$ diagrams for different ratios $H / G$, and show the regions corresponding to the different phases and the position of triple and end points. In the graphs, solid and dotted lines correspond to the mentioned first order and crossover transitions, respectively. Between NQM and 2SC regions we find that in all cases there is a second order phase transition, which corresponds to the dashed lines in the diagrams of Fig. 2. Close to this phase border, the dashed-dotted lines in the graphs delimit a band that corresponds to the so-called gapless $2 \mathrm{SC}$ (g2SC) phase. In this region, in addition to the two gapless modes corresponding to the unpaired blue quarks, the presence of flavor asymmetric chemical potentials $\mu_{d c}-\mu_{u c} \neq 0$ gives rise to another two gapless fermionic quasiparticles (the numerical determination of this phase border is somewhat subtle in these models, owing to the nonlocality of the interactions). In any case, for the range of parameters considered here, the $\mathrm{g} 2 \mathrm{SC}$ region is found to be too narrow to lead to sizeable effects.

\section{DISCUSSION}

Let us discuss some qualitative features of the curves displayed in Figs. 1 and 2. On one hand, for both models the 2SC phase region becomes larger when the ratio $H / G$ is increased. This is not surprising, since $H$ is the effective coupling governing the quark-quark interaction that gives rise to the pairing. As a general conclusion, it can be stated that, provided the ratio $H / G$ is not too low, the nonlocal schemes favor the existence of color superconducting phases at low temperatures and moderate chemical potentials (notice that, for the parameters considered here, we do not find color superconductivity only in the case of Model II with $H / G=0.5$ ). This is in contrast e.g. with the situation in the NJL model [5], where the existence of a 2SC phase turns out to be rather dependent on the input parameters. In addition, our results are qualitatively different from those obtained in the case of noncovariant nonlocal models [8], where above the chiral phase transition the NQM phase is preferable for values of the coupling ratio $H / G \lesssim 0.75$. In those models, a color superconducting quark matter phase can be found only for $H / G \approx 1$.

It is also interesting to compare our results with those obtained for isospin symmetric quark matter. For the same parameter sets, the corresponding phase diagrams for $H / G=0.75$ are shown in Fig. 3. By comparing them with those of Fig. 2, it can be seen that the $2 \mathrm{SC}$ region becomes reduced when one imposes color and electric charge neutrality conditions. This is indeed what one would expect, since the condition of electric charge neutrality leads in general to unequal $u$ and $d$ quark densities, disfavoring the $u$ - $d$ pairing. We notice, however, that the effect is relatively small, and the positions of triple and end points as well as the shape of the critical lines remain approximately unchanged.
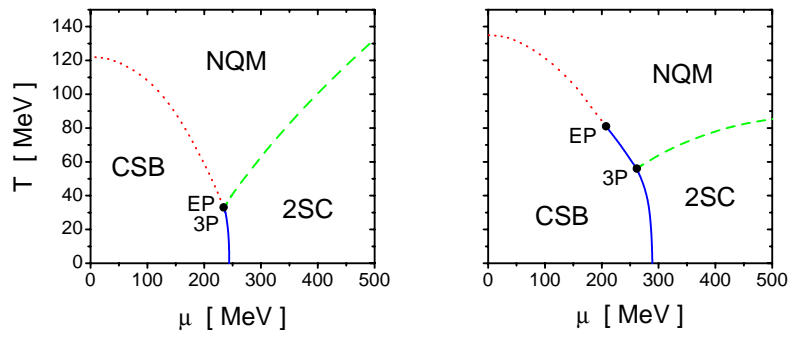

FIG. 3: Phase diagrams for symmetric matter corresponding to Models I (left) and II (right). Here we use $H / G=0.75$

Finally, we have studied the dependence of the phase tran- 
sitions on the model parameters, changing the input value of the chiral condensate within a phenomenologically reasonable range $220 \mathrm{MeV} \leq-\langle 0|\bar{q} q| 0\rangle^{1 / 3} \leq 280 \mathrm{MeV}$. From this analysis, it is seen that the qualitative features of the phase diagrams are not significantly modified. In particular, it is seen that one finds in general some sort of color superconducting phases at low temperatures and moderate chemical potentials, for intermediate values of the ratio $H / G$. In addition, the values for the critical temperature at $\mu=0$ are quite stable, yielding about $120 \mathrm{MeV}$ for Model I and $140 \mathrm{MeV}$ for Model II. This would favor the description given by Model II, in which the result is closer to the values provided by lattice calculations.

\section{CONCLUSIONS}

We have considered the phase diagram of two-flavor quark matter under neutron star constraints for two nonlocal, covariant quark models within the mean field approximation. In the first case the nonlocality was due to the regularization procedure, motivated by the instanton liquid model (Model I), whereas in the second model a separable approximation of the one-gluon exchange interaction was applied (Model II). Although for the Gaussian regulators considered in this work the vacuum gap equations are identical, both models differ in their fluctuation spectrum and therefore in their parameters (current quark mass $m$, coupling strength $G, \mathrm{UV}$ cutoff $\Lambda$ ) which have been fixed by the pion mass, the pion decay constant and the chiral condensate. As result of the numerical evaluation of the corresponding gap equations at finite tem- perature and chemical potential, we have obtained that Model II predicts a larger quark mass gap and a chiral symmetry breaking (CSB) phase transition line which extends 15-20\% further into the $T-\mu$ plane when compared to Model I. The prediction for the critical temperature at $\mu=0$ in Model II, $T_{\mathrm{CSB}} \sim 140 \mathrm{MeV}$, is closer to the results of recent lattice QCD simulations than the prediction of both Model I and the wellknown local NJL model. Considering different values for the coupling strength in the scalar diquark channel, we have found that under neutron star constraints different low-temperature quark matter phases can occur at intermediate densities: normal quark matter (NQM), pure superconducting (2SC) quark matter and mixed 2SC-NQM phases, respectively. The critical temperature for the $2 \mathrm{SC}$ phase transition is a rising function of $\mu$ for Model I whereas it is rather flat for Model II, due to the different $\mu$ dependences associated with the scalar diquark gaps. A band of gapless $2 \mathrm{SC}$ phase appears at the border of the superconducting region, but this occurs in general at nonzero temperatures and should not represent a robust feature for compact star applications.

\section{Acknowledgments}

This work has been supported in part by CONICET and ANPCyT (Argentina), under grants PIP 02368, PICT00-0308580 and PICT02-03-10718, and by a scientist exchange program between Germany and Argentina funded jointly by DAAD under grant No. DE/04/27956 and ANTORCHAS under grant No. 4248-6.
[1] E. Shuryak, J. Phys. G 30, S1221 (2004); Prog. Part. Nucl. Phys. 53, 273 (2004)

[2] D. Blaschke, N. K. . Glendenning, and A. Sedrakian (Eds.), "Physics of neutron star interiors", Springer, Lecture Notes in Physics 578 (2001).

[3] M. Alford, K. Rajagopal, and F. Wilczek, Phys. Lett. B 422, 247 (1998); R. Rapp, T. Schäfer, E.V. Shuryak, and M. Velkovsky, Phys. Rev. Lett. 81, 53 (1998)

[4] M. Alford and K. Rajagopal, JHEP 0206, 031 (2002)

[5] M. Buballa, Phys. Rept. 407, 205 (2005)

[6] S. B. Rüster, V. Werth, M. Buballa, I. A. Shovkovy, and D. H. Rischke, Phys. Rev. D 72, 034004 (2005); D. Blaschke, S. Fredriksson, H. Grigorian, A. M. Öztas, and F. Sandin, Phys. Rev. D 72, 065020 (2005)

[7] D. Blaschke, S. Fredriksson, H. Grigorian, and A. M. Öztas, Nucl. Phys. A 736, 203 (2004); H. Grigorian, D. Blaschke, and D. N. Aguilera, Phys. Rev. C 69, 065802 (2004)

[8] D. N. Aguilera, D. Blaschke, and H. Grigorian, Nucl. Phys. A 757, 527 (2005)

[9] T. Schafer and E.V. Schuryak, Rev. Mod. Phys. 70, 323 (1998)

[10] C. D. Roberts and A. G. Williams, Prog. Part. Nucl. Phys. 33, 477 (1994); C. D. Roberts and S. M. Schmidt, Prog. Part. Nucl. Phys. 45, S1 (2000)

[11] J. Skullerud, D. B. Leinweber, and A. G. Williams, Phys. Rev. D 64, 074508 (2001)

[12] U. Vogl and W. Weise, Prog. Part. Nucl. Phys. 27, 195 (1991); S. Klevansky, Rev. Mod. Phys. 64, 649 (1992); T. Hatsuda and
T. Kunihiro, Phys. Rep. 247, 221 (1994)

[13] E. Ruiz Arriola and L.L. Salcedo, Phys. Lett. B 450, 225 (1999)

[14] G. Ripka, Nucl. Phys. A 683, 463 (2001); R.S. Plant and M.C. Birse, Nucl. Phys. A 703, 717 (2002)

[15] H. Ito, W. Buck, and F. Gross, Phys. Rev. C 43, 2483 (1991); Phys. Rev. C 45, 1918 (1992);

[16] M. Buballa and S. Krewald, Phys. Lett. B 294, 19 (1992);

[17] R. D. Bowler and M.C. Birse, Nucl. Phys. A 582, 655 (1995); R.S. Plant and M.C. Birse, Nucl. Phys. A 628, 607 (1998)

[18] A. Scarpettini, D. Gomez Dumm, and N. N. Scoccola, Phys. Rev. D 69, 114018 (2004)

[19] W. Broniowski, B. Golli, and G. Ripka, Nucl. Phys. A 703, 667 (2002); A.H. Rezaeian, N.R. Walet, and M.C. Birse, Phys. Rev. C 70, 065203 (2004)

[20] I. General, D. Gómez Dumm, and N.N. Scoccola, Phys. Lett. B 506, 267 (2001); D. Gómez Dumm and N.N. Scoccola, Phys. Rev. D 65, 074021 (2002)

[21] D. Gómez Dumm and N.N. Scoccola, Phys. Rev. C 72, 014909 (2005)

[22] R. S. Duhau, A. G. Grunfeld, and N. N. Scoccola, Phys. Rev. D 70, 074026 (2004)

[23] D. Blaschke, H. Grigorian, A. Khalatyan, and D. N. Voskresensky, Nucl. Phys. Proc. Suppl. 141, 137 (2005)

[24] G. Ripka, Quarks bound by chiral fields (Oxford University Press, Oxford, 1997)

[25] S. M. Schmidt, D. Blaschke, and Y. L. Kalinovsky, Phys. Rev. C 50, 435 (1994) 